\title{
Viral Oncolytic Therapy
}

\author{
Omar Zurkiya ${ }^{1} \quad$ Suvranu Ganguli ${ }^{1}$ \\ ${ }^{1}$ Division of Interventional Radiology, Massachusetts General \\ Hospital, Harvard Medical School, Boston, Massachusetts, United \\ States
}

Address for correspondence Omar Zurkiya, MD, PhD, Massachusetts General Hospital, Harvard Medical School, 55 Fruit Street, Boston, MA 02114, United States (e-mail: OZURKIYA@mgh.harvard.edu).

J Clin Interv Radiol ISVIR 2017;1:96-99.

\begin{abstract}
Viral oncolysis broadly refers to the use of modified viruses to infect and subsequently lyse tumor cells. This concept arises from the observation that viral replication is itself effective in destroying tumor cells. This effect is then amplified by reinfection of adjacent tumor cells by the progeny virion released from lysed tumor cells. Herpes simplex virus 1 (HSV-1) has been the primary focus of current efforts in viral oncolysis. It is a double-stranded DNA virus that is a ubiquitous pathogen transmitted by direct mucosal contact. HSV-1 possesses several features well suited to viral oncolytic therapy. It does not integrate into the cellular genome, has a large transgene capacity of up to $50 \mathrm{~kb}$, and is already highly prevalent in the general population. In addition, effective antiherpetic agents are available to stop unwanted viral replication. HSV-1 mutants that preferentially replicate in neoplastic cells rather than normal cells have been characterized, and several variants of replication deficient HSV-1 mutants have been created and studied. They follow a common theme in that their replication is

Keywords

- viral oncolysis

- interventional oncology

- viral oncolytic therapy significantly attenuated in normal cells, while activated in cancer cells. Studies have been performed in various strains including those known as G207, NV1020, talimogene laherparepvec, and rRp450, and are reviewed here. Viral oncolysis is an exciting area of research with applications to tumors throughout the body. It holds promise as a new treatment for primary and metastatic liver cancer and may soon become a relevant therapy in interventional oncology.
\end{abstract}

\section{Introduction}

The liver is a common site for primary or metastatic disease. In addition to primary liver tumors, such as hepatocellular carcinoma, cholangiocarcinoma, and gallbladder carcinoma, several cancers are known to present with isolated hepatic metastasis, including colorectal carcinoma, ocular melanoma, and neuroendocrine cancers. Moreover, in patients with additional sites of extrahepatic disease, it is the hepatic disease burden that is often the cause of morbidity and mortality.

Liver resection, liver transplantation, and ablation remain the treatments of choice for prospects of cure; however, many patients are not ideal candidates due to number or size of lesions or other comorbidities. The response rate of primary liver tumors to chemotherapy is low.

Interventional oncology approaches including chemotherapy and radioembolization make use intra-arterial access to the liver for delivering therapy. Recent advances in oncolytic viruses, however, offer a new and promising intraarterial therapy.

Viral oncolysis broadly refers to the use of modified viruses to infect and subsequently lyse tumor cells. This concept arises from the observation that viral replication is itself effective in destroying tumor cells. This effect is then amplified by reinfection of adjacent tumor cells by the progeny virion released from lysed tumor cells.

Herpes simplex virus 1 (HSV-1) has been the primary focus of current efforts in viral oncolysis. ${ }^{1-4}$ It is a doublestranded DNA virus that is a ubiquitous pathogen transmitted by direct mucosal contact. Infection with HSV-1 is common, reported to be 66 to $84 \%$ in the United States. Skin or mucosal infection with HSV-1 is typically followed by transmission via sensory nerves to the trigeminal ganglia where lifelong latent infection occurs. Reactivation from ganglionic neurons may occur resulting in an epithelial ulcer
DOI https://doi.org/ $10.1055 / \mathrm{s}-0037-1602389$. ISSN $0000-0000$
Copyright ( 2017 by Indian Society of Vascular and Interventional Radiology
License terms

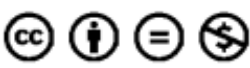


(e.g., "cold sore") with viral shedding into the oral cavity or epithelia in the trigeminal distribution.

HSV-1 possesses several features well suited to viral oncolytic therapy. It does not integrate into the cellular genome, has a large transgene capacity of up to $50 \mathrm{~kb}$, and is already highly prevalent in the general population. The presence of antibodies against HSV-1 does not attenuate its oncolytic efficacy. Despite the very high prevalence of exposure to HSV-1, it rarely causes severe illness, and effective antiherpetic agents are available to terminate unwanted viral replication.

HSV-1 mutants that preferentially replicate in neoplastic cells rather than normal cells have been characterized, and several variants of replication deficient HSV-1 mutants have been created and studied. They follow a common theme in that their replication is significantly attenuated in normal cells, while activated in cancer cells. Studies have been performed in various strains including those known as G207, NV1020, talimogene laherparepvec (T-VEC, formerly known as OncoVEX ${ }^{\mathrm{GM}-\mathrm{CSF}}$ ), and rRp450. Each of these mutants is attenuated relative to wild-type HSV-1 (-Table 1).

\section{Construction of the Viral Vector}

HSV is a neurotropic member of the human herpesvirus family. It is composed of two segments, a unique long $\left(\mathrm{U}_{\mathrm{L}}\right)$ and unique short $\left(U_{S}\right)$ segments with surrounding regulatory genes and elements. ${ }^{5}$ During a natural infection, the virus replicates in human skin or mucosa cells causing lysis of these cells. The released virions travel in neurons and continue along two main pathways of a replication cycle. They may continue to replicate in a lytic form or enter a latent state. The virus consists of a capsid and envelope with an intervening protein matrix. Replication-defective vectors are engineered by deleting one or more of the necessary HSV gene products. Several variants of replication deficient HSV1 mutants have been created and studied. These follow a similar theme in their construction in which the natural infection and replication of HSV-1 is attenuated in normal cells relative to cancer cells.

In the case of $\mathrm{rRp} 450$, a genetically engineered HSV-1 mutant of laboratory strain KOS created by Chase et al, ${ }^{1}$ the viral gene $\left(U_{L} 39\right)$ encoding infected cell protein 6 (ICP6) has

\section{Structure of RR - Mutant (rRp450)}

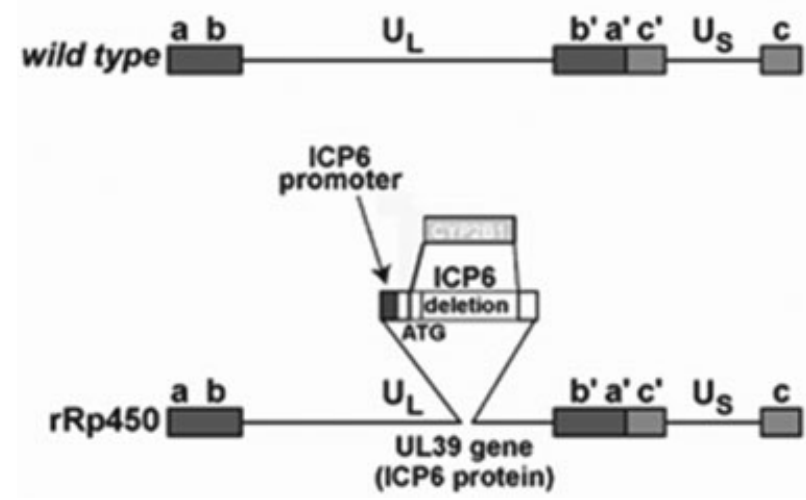

Fig. 1 Genomic structure of rRp450. The native HSV-1 repeats are shown as boxes (labeled $\mathrm{a}-\mathrm{c}$ ). The $U_{L} 39$ gene encoding the large subunit of the viral ribonucleotide reductase (ICP6) contains an internal deletion into which is cloned the rat cytochrome P450 (CYP2B1) gene under the control of the $U_{\mathrm{L}} 39$ (ICP6) promoter for ICP6. Several HSV-1 oncolytic viral vectors follow similar approaches in attenuating native viral replication. HSV-1, Herpes simplex virus 1; ICP6, infected cell protein 6.

been removed and substituted with the coding sequence of another gene, rat CYP2B1 cytochrome p450 gene ( - Fig. 1). rRp450 is, therefore, defective in expression of ICP6 (large subunit of viral ribonucleotide reductase), markedly attenuating its replication in normal cells. Its replication in cancer cells is significantly more robust, as these transformed cells provide nucleotide precursors to complement the absence of viral ribonucleotide reductase.

By virtue of the CYP2B1 transgene incorporated in rRp450, cells infected with rRp450 can also bioactivate the prodrug cyclophosphamide, leading to chemotherapeutic toxicity in addition to cytotoxicity caused by viral replication. This raises the potential of administering rRp450 together with cyclophosphamide for enhanced effect.

G207 is an HSV-1 mutant in which both copies of the $\gamma 34.5$ gene, implicated in viral replication have been deleted. lac $Z$ is also inserted in the $U_{L} 39$ gene to inactivate viral ribonucleotide reductase. T-VEC makes use of similar pathway which renders the virus incapable of replicating in normal cells.

Table 1 List of HSV-1-based oncolytic viruses in ongoing clinical evaluation

\begin{tabular}{|l|l|l|}
\hline Vector name & Clinical stage and indication & Method of delivery \\
\hline G207 & $\begin{array}{l}\text { Phase I for glioblastoma and other brain } \\
\text { tumors }\end{array}$ & Stereotactic injection into tumor \\
\hline NV1020 & $\begin{array}{l}\text { Phases I and II for metastatic colorectal } \\
\text { cancer to liver }\end{array}$ & Intravascular delivery to liver \\
\hline T-VEC (formerly known as OncoVex & $\begin{array}{l}\text { FDA-CSF) } \\
\text { melanomal in the United States for }\end{array}$ & Direct injection into tumor \\
\hline rRp450 & $\begin{array}{l}\text { Phase I for primary and metastatic } \\
\text { tumors to liver }\end{array}$ & Intravascular delivery to liver \\
\hline
\end{tabular}

Abbreviations: FDA, Food and Drug Administration; T-VEC, talimogene laherparepvec. 
The common theme is that interruption of essential gene products in virus replication results in HSV mutants incapable of replicating in normal cells. Cancer cells, however, in the process of transforming into malignant cells, have acquired deficiencies in antiviral mechanisms allowing these same viral mutants to retain the ability to replicate with resultant cellular lysis.

\section{Current State of Viral Oncolytic Therapy}

G207 is an HSV-1 mutant under development by MediGene in which both copies of the $\gamma 134.5$ gene, implicated in viral replication have been deleted, and the $U_{L} 39$ gene is inactivated. In a phase I trial establishing safety in subjects with brains tumors, no toxicity or serious adverse events were attributed to G207. ${ }^{6}$ In addition, there was no difference in side effects between patients who had previously been exposed to HSV-1 versus those not previously exposed. In a phase I trial from the University of Alabama, published in May 2014, ${ }^{7}$ G207 was administered stereotactically into glioblastoma tumors in conjunction with radiation. Six of the nine patients in the trial demonstrated stable disease or partial response for at least one of the time points in the study with three demonstrating marked radiographic response.

NV1020 is an HSV-1 mutant in which the internal repeat is deleted and replaced by a fragment of the HSV-2 genome. In a study of interest to the interventional radiology community, a phase I, open-label, dose-escalating trial in patients with metastatic colorectal carcinoma of the liver has been performed. Subjects received a single 10-minute hepatic arterial infusion of NV1020. ${ }^{8}$ Adverse events were either mild or moderate in severity and self-limiting. Only three serious adverse events (one transient rise in serum g-glutamyltransferase, one diarrhea, and one leukocytosis) experienced by three patients were considered to be possibly or probably related to NV1020. There was no evidence of disseminated herpes infection.

A multicenter phase I/II study published in September $2010^{2}$ evaluated 32 patients with advanced metastatic colorectal cancer of the liver. Patients received 4 weekly intraarterial NV1020 doses, followed by two or more cycles of conventional chemotherapy. An optimum biological dose of $10^{8}$ plaque forming units per dose was established. Of the 22 patients receiving this dose, 11 (50\%) initially showed stable disease.

T-VEC is an HSV-1 mutant expressing granulocyte macrophage colony-stimulating factor. In a phase II study, T-VEC was injected intratumorally into patients with stage IIIC or IV melanoma. ${ }^{9}$ Patients received an initial injection into 1 to 10 accessible tumors followed by a 3-week interval, then continued with injections every two weeks for a total of up to 24 injections. Both patients previously exposed and not previously exposed to HSV-1were included in the study. Response rates were similar in these two groups. The overall response rate by RECIST (Response Evaluation Criteria In Solid Tumors) was 26\% (eight with complete response and five with partial response). Overall survival was $58 \%$ at 1 year and $52 \%$ at 24 months.
T-VEC is currently being commercialized by Amgen, Inc. under the trade name Imlygic. As of October 2015, the U.S. Food and Drug Administration officially approved T-VEC for use in melanoma patients with injectable but nonresectable lesions in the skin and lymph nodes making it the first oncolytic virus approved for cancer therapy in the United States.

In separate studies, rRp450 is under investigation for viral oncolysis. Preclinical studies demonstrated that following administration into portal venous system, viral replication occurs preferentially in liver tumor cells versus normal liver. ${ }^{10}$ The cytotoxicity associated with the viral replication results in a marked reduction in tumor burden and prolongation of animal survival. Importantly, rRp450 retains its thymidine kinase gene, thereby rendering the virus susceptible to treatment with acyclovir and its analogs to terminate any unwanted viral replication.

A phase-I clinical trial to determine the safety of rRp450 and the highest dose of this agent that can be given safely is currently underway. This involves intra-arterial injection of rRp450 for treatment of primary liver tumors as well as metastatic tumors to the liver. Additional study goals include how the agent is absorbed by the liver cancers, how quickly it is eliminated, and evaluation of tumor response.

\section{Conclusion}

Viral oncolysis is an exciting area of research with applications to tumors throughout the body. Understanding viral oncolytic therapy background and development is important for future applications. For the interventional radiology community, intra-arterial viral oncolysis holds promise as a new treatment for primary and metastatic liver cancer and may soon become a relevant therapy in interventional oncology.

\section{References}

1 Chase M, Chung RY, Chiocca EA. An oncolytic viral mutant that delivers the CYP2B1 transgene and augments cyclophosphamide chemotherapy. Nat Biotechnol 1998;16(05):444-448

2 Geevarghese SK, Geller DA, de Haan HA, et al. Phase I/II study of oncolytic herpes simplex virus NV1020 in patients with extensively pretreated refractory colorectal cancer metastatic to the liver. Hum Gene Ther 2010;21(09):1119-1128

3 Kuruppu D, Tanabe KK. Viral oncolysis by herpes simplex virus and other viruses. Cancer Biol Ther 2005;4(05):524-531

4 Braidwood L, Graham SV, Graham A, Conner J. Oncolytic herpes viruses, chemotherapeutics, and other cancer drugs. Oncolytic Virother 2013;2:57-74

5 Goins WF, Huang S, Cohen JB, Glorioso JC. Engineering HSV-1 vectors for gene therapy. Methods Mol Biol 2014;1144:63-79

6 Markert JM, Medlock MD, Rabkin SD, et al. Conditionally replicating herpes simplex virus mutant, G207 for the treatment of malignant glioma: results of a phase I trial. Gene Ther 2000; 7(10):867-874

7 Jackson JD, McMorris AM, Roth JC, et al. Assessment of oncolytic HSV efficacy following increased entry-receptor expression in malignant peripheral nerve sheath tumor cell lines. Gene Ther 2014;21(11):984-990 
8 Kemeny N, Brown K, Covey A, et al. Phase I, open-label, doseescalating study of a genetically engineered herpes simplex virus, NV1020, in subjects with metastatic colorectal carcinoma to the liver. Hum Gene Ther 2006;17(12):1214-1224

9 Senzer NN, Kaufman HL, Amatruda T, et al. Phase II clinical trial of a granulocyte-macrophage colony-stimulating factor-encoding, second-generation oncolytic herpesvirus in patients with unresectable metastatic melanoma. J Clin Oncol 2009;27(34):5763-5771

10 Pawlik TM, Nakamura H, Yoon SS, et al. Oncolysis of diffuse hepatocellular carcinoma by intravascular administration of a replication-competent, genetically engineered herpesvirus. Cancer Res 2000;60(11):2790-2795 